\title{
A white noise approach to stochastic partial differential equations driven by the fractional Lévy noise
}

\section{Xuebin Lü ${ }^{1 *}$ (D) and Wanyang Dai ${ }^{2}$}

\author{
Correspondence: \\ Ivxuebin2008@163.com \\ 'Department of Applied \\ Mathematics, School of Physical and \\ Mathematical Sciences, Nanjing \\ Tech University, Nanjing, P.R. China \\ Full list of author information is \\ available at the end of the article
}

\begin{abstract}
In this paper, based on the white noise theory for $d$-parameter Lévy random fields given by (Holden et al. in Stochastic Partial Differential Equations: A modeling, white noise functional approach, 2010), we develop a white noise frame for anisotropic fractional Lévy random fields to solve the stochastic Poisson equation and the stochastic Schrödinger equation driven by the $d$-parameter fractional Lévy noise. The solutions for the two kinds of equations are all strong solutions given explicitly in the Lévy-Hida stochastic distribution space.
\end{abstract}

Keywords: White noise analysis; Anisotropic fractional Lévy noises; Stochastic Poisson equation; Stochastic Schrödinger equation

\section{Introduction}

In recent years, fractional Lévy processes are getting popular since they are more flexible in modeling the distributions of noises than fractional Brownian motions (FBMs). More precisely, they can capture large jumps and model high variability in the real systems appearing in finance, telecommunications, and so on. Meanwhile, they can also capture the long memory effect in a similar way as FBMs do (see $[1,6-9,12,13]$, etc.). Currently, more and more researchers have been attracted to the studies of fractional Lévy processes, stochastic calculus for fractional Lévy processes, and stochastic differential equations driven by these processes. In [14], the authors defined a stochastic integral for a class of deterministic integrands with respect to real-valued fractional Lévy processes. In [13], we defined a stochastic integral for a class of real deterministic functions and deterministic operator-valued processes with respect to fractional Lévy processes on Gel'fand triple. In [1], by using $S$ transform the authors investigated the Skorokhod integral for fractional Lévy processes whose underlying Lévy processes have finite moments of any order by avoiding Malliavin calculus and white noise analysis. In [11], a white noise theory for fractional Lévy process was developed by considering it as a generalized functional of the sample path of Lévy process and solving several kinds of stochastic ordinary differential equations driven by fractional Lévy noises.

The object of this paper is developing white noise theory for fractional Lévy random fields and then the study of stochastic partial differential equations (SPDEs) driven by a fractional Lévy noise, that is, solving stochastic Poisson and Schrödinger equations driven

(c) The Author(s) 2018. This article is distributed under the terms of the Creative Commons Attribution 4.0 International License (http://creativecommons.org/licenses/by/4.0/), which permits unrestricted use, distribution, and reproduction in any medium, provided you give appropriate credit to the original author(s) and the source, provide a link to the Creative Commons license, and indicate if changes were made. 
by a $d$-parameter fractional Lévy noise. The white noise theory for fractional Lévy random fields is based on the results of white noise theory for $d$-parameter Lévy random fields given by [5]. The technique used in solving our SPDEs is based on the method developed in $[5,10]$, and so on, where the SPDEs are driven by Guassian and Lévy white noises. For convenience, we introduce a generalized approach for our study as follows. Consider an SPDE driven by fractional Lévy noise and expressed by

$$
A\left(t, x, \partial_{t}, \nabla_{x}, U, \omega\right)=0
$$

where $A$ is a given function, $U=U(t, x, \omega)$ is an unknown random field, $\partial_{t}=\frac{\partial}{\partial t}$, and $\nabla_{x}=\left(\frac{\partial}{\partial x_{1}}, \ldots, \frac{\partial}{\partial x_{1}}\right)$. Note that here we interpret all products as Wick products and all functions as their versions through replacing the existing driving noise by the fractional Lévy noise, which can be considered as generalized Lévy-Hida stochastic distribution functions. Then we have

$$
A^{\diamond}\left(t, x, \partial_{t}, \nabla_{x}, U, \omega\right)=0 .
$$

We take the Hermite transform of (1.2), which turns Wick products into ordinary products (between possibly complex numbers). Then the equation takes the form

$$
\widetilde{A}\left(t, x, \partial_{t}, \nabla_{x}, \widetilde{U}, \tau\right)=0,
$$

where $\widetilde{U}=\mathcal{H} U$ is the Lévy-Hermite transform, and $\tau=\left(\tau_{1}, \tau_{2}, \ldots\right)$ is a complex sequence. Suppose we can find a solution $u=u(t, x, \tau)$ of the equation

$$
\widetilde{A}\left(t, x, \partial_{t}, \nabla_{x}, u, \tau\right)=0
$$

for each $\tau \in K_{q}(R)$ for some $q$, $R$, where $K_{q}(R)$ is defined on page 6 . Then, under certain conditions, we can take the inverse Lévy-Hermite transform $\widetilde{U}=\mathcal{H}^{-1} u$ and thereby obtain a solution of the original (Wick) equation. In fact, this method can be also applied to other kinds of SPDEs driven by fractional Lévy noises. Here we just investigate the Poisson and Schrödinger types just because they all involve the Laplace operator and contain no firstorder derivatives.

The remainder of this paper is organized as follows. In Sect. 2, we briefly recall the basic results on the white noise analysis for a $d$-parameter Lévy random field given by [5]. In Sect. 3, we define the formal derivative of a fractional Lévy random field as the fractional Lévy noise $\dot{X}^{\bar{\beta}}(x)$. Then by using the Wick product we define the Skorokhod integral with respect to fractional Lévy random field:

$$
\int_{\mathbb{R}^{d}} F(x) \delta X^{\bar{\beta}}(x):=\int_{\mathbb{R}^{d}} F(x) \diamond \dot{X}^{\bar{\beta}}(x) d x .
$$

Furthermore, we investigate the integrable conditions for $F$. In Sects. 4 and 5, based on the white noise theory developed in Sects. 2 and 3, we respectively solve the stochastic Poisson and Schrödinger equations driven by the $d$-parameter fractional Lévy noise, and the solutions are obtained in the strong sense in the Lévy-Hida stochastic distribution space. 


\section{White noise analysis for a Lévy random field}

In this section, we briefly review the white noise analysis for a Lévy random field given by $[5,10]$, and [15] for convenience of the reader and citations in the subsequent discussion.

Denote by $\mathcal{S}\left(\mathbb{R}^{d}\right)$ the Schwartz space of rapidly decreasing $C^{\infty}$-functions on $\mathbb{R}^{d}$ and by $\mathcal{S}^{\prime}\left(\mathbb{R}^{d}\right)$ the space of tempered distributions, let $\Omega=\mathcal{S}^{\prime}\left(\mathbb{R}^{d}\right), \mathcal{F}=B\left(\mathcal{S}^{\prime}\left(\mathbb{R}^{d}\right)\right)$ be the Borel $\sigma$ algebra on $\mathcal{S}^{\prime}\left(\mathbb{R}^{d}\right)$. By the Bochner-Minlos theorem there exists a probability $P$ on $\mathcal{S}^{\prime}\left(\mathbb{R}^{d}\right)$ such that

$$
\int_{\Omega} e^{i u\langle\omega, f\rangle} P(d \omega)=\exp \left\{\int_{\mathbb{R}^{d}} \psi(u f(y)) d y\right\}, \quad u \in \mathbb{R}, f \in \mathcal{S}\left(\mathbb{R}^{d}\right)
$$

where $\langle\omega, f\rangle$ denotes the action of $\omega \in \mathcal{S}^{\prime}\left(\mathbb{R}^{d}\right)$ on $f \in \mathcal{S}\left(\mathbb{R}^{d}\right)$,

$$
\psi(u)=\exp \left\{\int_{\mathbb{R}}\left[e^{i u z}-1-i u z\right] d \nu(z)\right\}, \quad u \in \mathbb{R},
$$

$v$ is the Lévy measure satisfying $v(\{0\})=0$ and $\int_{\mathbb{R}_{0}}\left(|z|^{2} \wedge 1\right) d v(z)<+\infty$, and $\mathbb{R}_{0}=\mathbb{R} \backslash\{0\}$. Moreover, we assume that

$$
\int_{|z|>1}|z|^{2} d v(z)<+\infty
$$

For $f \in \mathcal{S}\left(\mathbb{R}^{d}\right)$, define $\dot{X}(f)(\omega):=\langle\omega, f\rangle$. Then by (2.1) we have

$$
\begin{aligned}
& \mathbb{E}[\dot{X}(f)]=0, \\
& \mathbb{E}[\dot{X}(f)]^{2}=\int_{\mathbb{R}^{d}} f^{2}(y) d y \int_{\mathbb{R}_{0}} z^{2} d v(z) .
\end{aligned}
$$

We can extend the definition of $\dot{X}(f)(\omega)$ for $f \in \mathcal{S}\left(\mathbb{R}^{d}\right)$ to any $f \in L^{2}\left(\mathbb{R}^{d}\right)$ by choosing $f_{n} \in$ $\mathcal{S}\left(\mathbb{R}^{d}\right)$ such that $f_{n} \rightarrow f$ in $L^{2}\left(\mathbb{R}^{d}\right)$ and defining $\dot{X}(f)(\omega):=\lim _{n \rightarrow \infty} \dot{X}\left(f_{n}\right)(\omega)\left(\right.$ in $\left.L^{2}(P)\right)$.

For $x=\left(x_{1}, \ldots, x_{d}\right)$, define $\eta(x):=\dot{X}\left(1_{[0, x]}(\cdot)\right)$, where

$$
1_{[0, x]}(y)=\prod_{i=1}^{d} 1_{\left[0, x_{i}\right]}\left(y_{i}\right), \quad y=\left(y_{1}, \ldots, y_{d}\right)
$$

with

$$
1_{\left[0, x_{i}\right]}\left(y_{i}\right)= \begin{cases}1, & 0<y_{i}<x_{i} \text { or } x_{i}<y_{i}<0 \\ 0 & \text { otherwise. }\end{cases}
$$

The stochastic process $\left\{\eta(x), x \in \mathbb{R}^{d}\right\}$ has a càdlàg version, denoted by $X$. This process $\left\{X(x), x \in \mathbb{R}^{d}\right\}$ is a pure jump Lévy field with Lévy measure $\nu$. The process $X$ admits the stochastic integral representation

$$
X(x)=\int_{0}^{x} \int_{\mathbb{R}_{0}} z \tilde{N}(d y, d z)=\int_{0}^{x_{d}} \int_{0}^{x_{d-1}} \cdots \int_{0}^{x_{1}} \int_{\mathbb{R}_{0}} z \tilde{N}(d y, d z),
$$


where $N((0, x] \times A)=\sum_{y \in(0, x]} 1_{A}(\triangle X(y)), A \in \mathcal{B}(\mathbb{R})$ is a Poisson random measure on $\mathbb{R}^{d} \times$ $\mathbb{R}_{0}$, and $\widetilde{N}((0, x] \times A)=N((0, x] \times A)-\prod_{i=1}^{d} x_{i} v(A)$ is its compensator. In this case, $X$ is a pure-jump Lévy martingale.

Let $f=f\left(x^{1}, z_{1}, \ldots, x^{n}, z_{n}\right)$ be a symmetric function that is invariant under all permutations of the index set $\{1,2, \ldots, n\}$. If $f \in L^{2}\left(\left(\mathbb{R}^{d} \times \mathbb{R}\right)^{n},(\lambda \times v)^{n}\right)$, where $\lambda$ is the Lebesgue measure on $\mathbb{R}^{d}$, then define

$$
I_{n}(f):=n ! J_{n}(f),
$$

where

$$
J_{n}(f)=\int_{G_{n}} f\left(x^{1}, z_{1}, \ldots, x^{n}, z_{n}\right) \tilde{N}\left(d x^{1}, d z_{1}\right) \cdots \tilde{N}\left(d x^{n}, d z_{n}\right)
$$

with $G_{n}=\left\{\left(x^{1}, z_{1}, \ldots, x^{n}, z_{n}\right) \in\left(\mathbb{R}^{d} \times \mathbb{R}\right)^{n}: x_{j}^{1} \leq x_{j}^{2} \leq \cdots \leq x_{j}^{n}, \forall 1 \leq j \leq d\right\}$.

From now on we assume that the Lévy measure $v$ is such that, for any constant $\varepsilon>0$, there exists a constant $\lambda>0$ such that

$$
\int_{\mathbb{R}_{0} \backslash(-\epsilon, \epsilon)} \exp (\lambda|x|) d v(x)<+\infty
$$

Property (2.5) implies that the polynomials are dense in $L^{2}(\rho)$, where $\rho(d z)=z^{2} v(d z)$ (see[15]). Let $\left\{l_{0}, l_{1}, l_{2}, \ldots\right\}$ be the orthogonalization of $\left\{1, z, z^{2}, \ldots\right\}$ with respect to the inner product of $L^{2}(\rho)$. Define

$$
p_{j}(z):=\left\|l_{j-1}\right\|_{L^{2}(\rho)}^{-1} z l_{j-1}(z), \quad j=1,2, \ldots
$$

In particular, $p_{1}(x)=m_{2}^{-1} x$, where $m_{2}=\left(\int_{\mathbb{R}_{0}} z^{2} d v(z)\right)^{\frac{1}{2}}$. Then $\left\{p_{j}(x), j \geq 1\right\}$ is an orthonormal basis for $L^{2}(v)$.

Let $h: \mathbb{N}^{d} \rightarrow \mathbb{N}$ be a bijective map, where $\mathbb{N}^{d}$ is the $d$-fold Cartesian product of $\mathbb{N}=$ $\{1,2, \ldots\}$ such that if $i=h\left(i_{1}, \ldots, i_{d}\right)$, then $i_{k} \leq i$ for $k=1, \ldots, d$. As for the existence of such $h$, for $d=3$, define $\kappa(i, j)=j+\frac{(i+j-2)(i+j-1)}{2}$, which is a bijective map from $\mathbb{N}^{2}$ to $\mathbb{N}$ such that $i, j \leq \kappa(i, j)$; then define $i=h\left(i_{1}, i_{2}, i_{3}\right)=\kappa\left(\kappa\left(i_{1}, i_{2}\right), i_{3}\right)$. It is easy to see that $h$ is a bijective map $\mathbb{N}^{3} \rightarrow \mathbb{N}$ satisfying the demand. In general, we can define $h$ by induction in $d$.

If $i=h\left(i_{1}, \ldots, i_{d}\right)$, then define $\zeta_{i}(x)=\xi_{i_{1}}\left(x_{1}\right) \cdots \xi_{i_{d}}\left(x_{d}\right)$ for $x=\left(x_{1}, \ldots, x_{d}\right)$, where $\left\{\xi_{i}, i \geq 1\right\}$ are the Hermite functions. Then $\left\{\zeta_{i}(x), i \geq 1\right\}$ is an orthogonal basis of $L^{2}\left(\mathbb{R}^{d}\right)$.

Let $\delta_{\kappa(i, j)}(x, z)=\zeta_{i}(x) p_{j}(z)$, let $\mathcal{J}$ be the set of all sequences $\alpha=\left(\alpha_{1}, \alpha_{2}, \ldots, \alpha_{i}, \ldots\right)$ with $\alpha_{i} \in\{0,1,2, \ldots\}$ for all $i$ and with finitely $\alpha_{i} \neq 0$, and define index $(\alpha):=\max \left\{i: \alpha_{i} \neq 0\right\},|\alpha|:=$ $\sum_{i} \alpha_{i}, \alpha !:=\prod_{i} \alpha_{i}$ ! where $0 !=1$. For $\alpha \in \mathcal{J}$ with index $(\alpha)=j,|\alpha|=m$, we define

$$
\begin{aligned}
\delta^{\otimes \alpha} & \left(x^{1}, z_{1}, \ldots, x^{m}, z_{m}\right) \\
& =\delta_{1}^{\otimes \alpha_{1}} \otimes \cdots \otimes \delta_{j}^{\otimes \alpha_{j}}\left(x^{1}, z_{1}, \ldots, x^{m}, z_{m}\right) \\
& =\underbrace{\delta_{1}\left(x^{1}, z_{1}\right) \cdots \delta_{1}\left(x^{\alpha_{1}}, z_{\alpha_{1}}\right)}_{\alpha_{1} \text { factors }} \cdots \underbrace{\delta_{j}\left(x^{m-\alpha_{j}+1}, z_{m-\alpha_{j}+1}\right) \cdots \delta_{j}\left(x^{m}, z_{m}\right)}_{\alpha_{j} \text { factors }},
\end{aligned}
$$


where $\otimes$ is the tensor product. We set $\delta_{j}^{\otimes 0}=1$ and $\delta^{\widehat{\otimes} \alpha}\left(x^{1}, z_{1}, \ldots, x^{m}, z_{m}\right):=\delta_{1}^{\widehat{\otimes} \alpha_{1}} \otimes \cdots \otimes$ $\delta_{j}^{\widehat{\otimes} \alpha_{j}}\left(x^{1}, z_{1}, \ldots, x^{m}, z_{m}\right)$, where $\widehat{\otimes}$ is the symmetric tensor product, and define

$$
K_{\alpha}:=I_{|\alpha|}\left(\delta^{\widehat{\otimes} \alpha}\right) .
$$

Theorem 2.1 ([5]) Any $F \in L^{2}(P)$ has a unique expansion of the form

$$
F=\sum_{\alpha \in \mathcal{J}} c_{\alpha} K_{\alpha}
$$

with $c_{\alpha} \in \mathbb{R}$. Moreover,

$$
\|F\|_{L^{2}(P)}^{2}=\sum_{\alpha \in \mathcal{J}} \alpha ! c_{\alpha}^{2}
$$

By Theorem 2.1, for $h \in L^{2}\left(\mathbb{R}^{d}\right)$,

$$
\dot{X}(h)=\int_{\mathbb{R}^{d}} h(x) d X(x)=m_{2} \sum_{i \geq 1} \int_{\mathbb{R}^{d}} h(x) \zeta_{i}(x) d x K_{\varepsilon(i, 1)},
$$

where $K_{\varepsilon(i, 1)}=I_{1}\left(\xi_{i}(x) p_{1}(z)\right), \varepsilon(i, 1)=(0,0, \ldots, 1,0, \ldots)$ with the 1 on the $\kappa(i, 1)$ th place.

Definition 2.2 (The Lévy-Hida spaces, $[5,10])$

(1) Stochastic test functions $(\mathcal{S})$

Let $(\mathcal{S})$ consist of all $\varphi=\sum_{\alpha \in \mathcal{J}} c_{\alpha} K_{\alpha}$ such that

$$
\|\varphi\|_{k}^{2}:=\sum_{\alpha \in \mathcal{J}} c_{\alpha}^{2}(\alpha !)^{2}(2 \mathbb{N})^{k \alpha}<+\infty \quad \text { for all } k \in \mathbb{N},
$$

equipped with the projective topology, where

$$
(2 \mathbb{N})^{k \alpha}=\prod_{j \geq 1}(2 j)^{k \alpha_{j}} .
$$

(2) Stochastic distribution $(\mathcal{S})^{-1}$

Let $\mathcal{S}^{-p}$ consist of all $\varphi=\sum_{\alpha \in \mathcal{J}} c_{\alpha} K_{\alpha}$ such that

$$
\|\varphi\|_{-p}^{2}:=\sum_{\alpha \in \mathcal{J}} c_{\alpha}^{2} \alpha !(2 \mathbb{N})^{-p \alpha}<+\infty
$$

and define $(\mathcal{S})^{-1}$ as the inductive limit of $\left\{\mathcal{S}^{-p}, p \in \mathbb{N}\right\},(\mathcal{S})^{-1}$ is the dual of $(\mathcal{S})$. If $F=$ $\sum_{\alpha \in \mathcal{J}} a_{\alpha} K_{\alpha} \in(\mathcal{S})^{-1}$ and $\varphi=\sum_{\alpha \in \mathcal{J}} b_{\alpha} K_{\alpha} \in(\mathcal{S})$, then the action of $F$ on $\varphi$ is

$$
\langle\langle F, \varphi\rangle\rangle=\sum_{\alpha \in \mathcal{J}} a_{\alpha} b_{\alpha} \alpha !
$$

Definition 2.3 (Wick product, [10]) If $F=\sum_{\alpha \in \mathcal{J}} a_{\alpha} K_{\alpha} \in(\mathcal{S})^{-1}$ and $G=\sum_{\alpha \in \mathcal{J}} b_{\alpha} K_{\alpha} \in$ $(\mathcal{S})^{-1}$, then the Wick product of $F$ and $G$ is defined as

$$
F \diamond G:=\sum_{\alpha, \beta \in \mathcal{J}} a_{\alpha} b_{\beta} K_{\alpha+\beta} .
$$


More generally,

$$
\begin{aligned}
& F^{\diamond n}:=\underbrace{F \diamond F \diamond \cdots \diamond F}_{n \text { times }}, \\
& \exp ^{\diamond} F:=\sum_{n=0}^{\infty} \frac{F^{\diamond n}}{n !} .
\end{aligned}
$$

Proposition 2.4 Suppose $F=\sum_{\alpha \in \mathcal{J}} a_{\alpha} K_{\alpha} \in \mathcal{S}^{-k}$ and $G=\sum_{\alpha \in \mathcal{J}} b_{\alpha} K_{\alpha} \in \mathcal{S}^{-l}$ with $k>l+1$.

Then

$$
\|F \diamond G\|_{-k} \leq A(k-l)\|F\|_{-k}\|G\|_{-l}
$$

where

$$
A(k-l)=\sum_{\alpha \in \mathcal{J}}(2 \mathbb{N})^{(l-k) \alpha}<+\infty .
$$

Proof Analogous to the proof of Proposition 3.3.2 in [5].

Definition 2.5 ([10]) If $F=\sum_{\alpha \in \mathcal{J}} a_{\alpha} K_{\alpha} \in(\mathcal{S})^{-1}$, then the Lévy-Hermite transform of $F$ is defined by

$$
\mathcal{H} F(\tau):=\sum_{\alpha \in \mathcal{J}} a_{\alpha} \tau^{\alpha}=\sum_{\alpha \in \mathcal{J}} a_{\alpha} \prod_{k} \tau_{k}^{\alpha_{k}}
$$

for $\tau=\left(\tau_{1}, \tau_{2}, \ldots\right) \in \mathbb{C}^{\mathbb{N}}$, where $\mathbb{C}$ is the complex plane, and $\mathbb{C}^{\mathbb{N}}$ is the set of all sequences $\tau=\left(\tau_{1}, \tau_{2}, \ldots\right)$ with $\tau_{i} \in \mathbb{C}$.

An important property of the Lévy-Hermite transform is that it transforms the Wick product into an ordinary product, that is,

$$
\mathcal{H}(F \diamond G)(\tau)=\mathcal{H} F(\tau) \mathcal{H} G(\tau) .
$$

For $0<R<+\infty, q \in \mathbb{N}$, define

$$
K_{q}(R):=\left\{\tau=\left(\tau_{1}, \tau_{2}, \ldots\right) \in \mathbb{C}^{\mathbb{N}}: \sum_{\alpha \neq 0}\left|\tau^{\alpha}\right|^{2}(2 \mathbb{N})^{q \alpha}<R^{2}\right\} .
$$

In [10], the following characterization theorem for the stochastic distribution $(\mathcal{S})^{-1}$ is given.

Theorem 2.6 ([10])

(1) If $F=\sum_{\alpha \in \mathcal{J}} a_{\alpha} K_{\alpha} \in(\mathcal{S})^{-1}$, then there exist $q \in \mathbb{N}$ and $0<M_{q}<+\infty$ such that

$$
|\mathcal{H} F(z)| \leq \sum_{\alpha}\left|a_{\alpha}\right|\left|\tau^{\alpha}\right| \leq M_{q}\left(\sum_{\alpha \neq 0}\left|\tau^{\alpha}\right|^{2}(2 \mathbb{N})^{q \alpha}\right)^{\frac{1}{2}}
$$

for all $\tau \in\left(\mathbb{C}^{\mathbb{N}}\right)_{C}$, where $\left(\mathbb{C}^{\mathbb{N}}\right)_{C}$ is the set of all finite sequences in $\mathbb{C}^{\mathbb{N}}$.

In particular, $\mathcal{H} F$ is a bounded analytic function on $K_{q}(R)$ for all $0<R<+\infty$. 
(2) Conversely, assume that $f(\tau)=\sum_{\alpha} a_{\alpha} \tau^{\alpha}$ is absolutely convergent and bounded on $K_{q}(R)$ for some $R>0$ and $0<q<\infty$. Then there exists a unique $F \in(\mathcal{S})^{-1}$ such that $\mathcal{H} F=f$ and

$$
F=\sum_{\alpha \in \mathcal{J}} a_{\alpha} K_{\alpha}
$$

Definition $2.7([5])$ Suppose $F: \mathbb{R}^{d} \longrightarrow(\mathcal{S})^{-1}$ is a given function such that $\langle\langle F(x), f\rangle\rangle \in$ $L^{1}\left(\mathbb{R}^{d}, d x\right)$ for all $f \in \mathcal{S}$. Then $\int_{\mathbb{R}^{d}} F(x) d x$ is defined to be the unique element of $(\mathcal{S})^{-1}$ such that

$$
\left\langle\left\langle\int_{\mathbb{R}^{d}} F(x) d x, f\right\rangle\right\rangle=\int_{\mathbb{R}^{d}}\langle\langle F(x), f\rangle\rangle d x .
$$

Lemma 2.8 Let $F(x)=\sum_{\alpha \in \mathcal{J}} c_{\alpha}(x) K_{\alpha}$ with

$$
\sum_{\alpha \in \mathcal{J}}\left\|c_{\alpha}(\cdot)\right\|_{L^{1}\left(\mathbb{R}^{d}\right)}^{2}(2 \mathbb{N})^{-p \alpha}<+\infty
$$

for some $p \in \mathbb{N}$. Then $F$ is $(\mathcal{S})^{-1}$-integrable, and

$$
\int_{\mathbb{R}^{d}} F(x) d x=\sum_{\alpha \in \mathcal{J}} \int_{\mathbb{R}^{d}} c_{\alpha}(x) d x K_{\alpha} .
$$

Proof Analogous to the proof of Lemma 2.5.6 in [5].

Theorem 2.9 ([5]) Suppose $u=u(t, x, \tau)$ is a solution (usually in strong pointwise sense) of the equation

$$
\widetilde{A}\left(t, x, \partial_{t}, \nabla_{x}, u, \tau\right)=0
$$

for all $(t, x)$ in some bounded open set $G \subset \mathbb{R} \times \mathbb{R}^{d}$ and for all $\tau \in K_{q}(R)$ for some $q, R$. Moreover, suppose that $u=u(t, x, \tau)$ and all its partial derivatives involved in (2.9) are bounded on $(x, \tau) \in D \times K_{q}(R)$, continuous with respect to $x \in D$ for each $\tau \in K_{q}(R)$, and analytic with respect to $\tau \in K_{q}(R)$ for all $x \in D$. Then there exists $U(t, x) \in(\mathcal{S})^{-1}$ such that $u(t, x, \tau)=(\mathcal{H} U(t, x))(\tau)$ for all $(t, x, \tau) \in G \times K_{q}(R)$ and $U(t, x)$ solves (in the strong sense in $\left.(\mathcal{S})^{-1}\right)$ the equation

$$
A^{\diamond}\left(t, x, \partial_{t}, \nabla_{x}, U, \omega\right)=0
$$

\section{White noise analysis for an anisotropic fractional Lévy random field}

In this section, according to the result of Sect. 2, we give the white noise analysis for the anisotropic fractional Lévy random field. First, we give its chaos expansion by considering it as a generalized functional of the path of the Lévy random field. Second, based on the chaos expansion of the anisotropic fractional Lévy random field, we define its formal derivative as a $d$-parameter fractional Lévy noise. Finally, we define the stochastic integration and give an integrability condition. 
Let $0<\beta<\frac{1}{2}$ and $f \in \mathcal{S}(\mathbb{R})$. The Riemann-Liouville fractional integral operator $I_{ \pm}^{\beta}$ is defined by

$$
\begin{aligned}
& \left(I_{-}^{\beta} f\right)(t)=\frac{1}{\Gamma(\beta)} \int_{t}^{+\infty}(s-t)^{\beta-1} f(s) d s, \\
& \left(I_{+}^{\beta} f\right)(t)=\frac{1}{\Gamma(\beta)} \int_{-\infty}^{t}(t-s)^{\beta-1} f(s) d s,
\end{aligned}
$$

where $\Gamma$ is the gamma function. (For more detail about the Riemann-Liouville fractional integral operator, see[16].)

Definition 3.1 For $\bar{\beta}=\left(\beta_{1}, \ldots, \beta_{d}\right), 0<\beta_{k}<\frac{1}{2}, k=1,2, \ldots, d$, the anisotropic fractional Lévy random field is defined by

$$
X^{\bar{\beta}}(x):=\dot{X}\left(I_{-\ldots-}^{\bar{\beta}} \chi_{[0, x]}\right)=\int_{\mathbb{R}^{d}} I_{-\ldots-}^{\bar{\beta}} \chi_{[0, x]}(y) d X(y), \quad x \in \mathbb{R}^{d},
$$

where $I_{-\ldots-}^{\bar{\beta}} \chi_{[0, x]}(y)=\prod_{k=1}^{d} I_{-}^{\beta_{k}} \chi_{\left[0, x_{k}\right]}\left(y_{k}\right)$

$$
\chi_{\left[0, x_{i}\right]}\left(y_{i}\right)= \begin{cases}1, & 0<y_{i}<x_{i} \\ -1, & x_{i}<y_{i}<0 \\ 0 & \text { otherwise }\end{cases}
$$

Note that $I_{-\ldots-}^{\bar{\beta}} \chi_{[0, x]}(\cdot) \in L^{2}\left(\mathbb{R}^{d}\right), X^{\bar{\beta}}(x)$ is well defined. The field (3.3) can be represented as

$$
X^{\bar{\beta}}(x)=\frac{1}{\Gamma(\bar{\beta}+1)} \int_{-\infty}^{x_{1}} \ldots \int_{-\infty}^{x_{d}} \prod_{k=1}^{d}\left[\left(x_{k}-y_{k}\right)^{\beta_{k}}-\left(y_{k}\right)_{+}^{\beta_{k}}\right] d X(y),
$$

where $x_{+}=\max \{x, 0\}$ and $\Gamma(\bar{\beta})=\prod_{k=1}^{d} \Gamma\left(\beta_{k}+1\right)$. From (3.4) we see that the fractional integral parameters along different time axes are different. Thus, the fractional Lévy random field $\left\{X^{\bar{\beta}}(x), x \in \mathbb{R}^{d}\right\}$ is anisotropic. By (2.6), $X^{\bar{\beta}}(x)$ has the chaos representation

$$
X^{\bar{\beta}}(x)=m_{2} \sum_{i \geq 1} \int_{\mathbb{R}^{d}} I_{-\ldots-}^{\bar{\beta}} \chi_{[0, x]}(y) \zeta_{i}(y) d y K_{\varepsilon(i, 1)} .
$$

Thus, by (2.7) we get the Lévy-Hermite transform of the anisotropic fractional Lévy random field

$$
\mathcal{H} X^{\bar{\beta}}(x)(\tau)=m_{2} \sum_{i \geq 1} \int_{\mathbb{R}^{d}} I_{-\ldots-\ldots}^{\bar{\beta}} \chi_{[0, x]}(y) \zeta_{i}(y) d y \tau_{\varepsilon(i, 1)}
$$

On the other hand, by the fractional integral by parts formula of the operator $I_{ \pm}^{\beta}$,

$$
\int_{\mathbb{R}} f(x) I_{+}^{\beta} g(x) d x=\int_{\mathbb{R}} g(x) I_{-}^{\beta} f(x) d x, \quad f, g \in \mathcal{S}(\mathbb{R})
$$


which can be extended to $f \in L^{p}(\mathbb{R})$ and $g \in L^{r}(\mathbb{R})$ with $p>1, r>1$, and $\frac{1}{p}+\frac{1}{r}=1+\beta$ (see[16]), (3.5) can be written as

$$
\begin{aligned}
X^{\bar{\beta}}(x) & =m_{2} \sum_{i \geq 1} \int_{\mathbb{R}^{d}} I_{-\ldots-}^{\bar{\beta}} \chi_{[0, x]}(y) \zeta_{i}(y) d y K_{\varepsilon(i, 1)} \\
& =m_{2} \sum_{i \geq 1, h\left(i_{1}, \ldots, i_{d}\right)=i} \prod_{k=1}^{d} \int_{\mathbb{R}} I_{-}^{\beta_{k}} \chi_{\left[0, x_{k}\right]}\left(y_{k}\right) \xi_{i_{k}}\left(y_{k}\right) d y_{k} K_{\varepsilon(i, 1)} \\
& =m_{2} \sum_{i \geq 1, h\left(i_{1}, \ldots, i_{d}\right)=i} \prod_{k=1}^{d} \int_{\mathbb{R}} \chi_{\left[0, x_{k}\right]}\left(y_{k}\right) I_{+}^{\beta_{k}} \xi_{i_{k}}\left(y_{k}\right) d y_{k} K_{\varepsilon(i, 1)} \\
& =m_{2} \sum_{i \geq 1} \int_{0}^{x_{1}} \ldots \int_{0}^{x_{d}} I_{+\ldots+}^{\bar{\beta}} \zeta_{i}(y) d y K_{\varepsilon(i, 1)},
\end{aligned}
$$

where $I_{+\cdots+}^{\bar{\beta}} \zeta_{i}(y):=\prod_{k=1}^{d} I_{+}^{\beta_{k}} \xi_{i_{k}}\left(y_{k}\right), h\left(i_{1}, \ldots, i_{d}\right)=i$. We denote by $\dot{X}^{\bar{\beta}}(x)$ the fractional Lévy noise in the following sense:

$$
\dot{X}^{\bar{\beta}}(x):=\frac{\partial^{d}}{\partial x_{1} \cdots \partial x_{d}} X^{\bar{\beta}}(x)=m_{2} \sum_{i \geq 1} I_{+\cdots+}^{\bar{\beta}} \zeta_{i}(x) K_{\varepsilon(i, 1)}
$$

Next, we prove that $\dot{X}^{\bar{\beta}}(x)$ is a generalized stochastic distribution function.

Theorem $3.2 \dot{X}^{\bar{\beta}}(x) \in \mathcal{S}^{-p}$ for all $p>2$.

Proof We will use the estimate

$$
\int_{\mathbb{R}}(t-u)_{+}^{\beta-1} \xi_{n}(u) d u \leq C n^{\frac{2}{3}-\frac{\beta}{2}}
$$

from Sect. 4 of [4], where $C$ is a certain positive constant independent of $t$. From now on, we denote by $C$ all positive constants. We have

$$
\begin{aligned}
\left\|\dot{X}^{\bar{\beta}}(x)\right\|_{-p}^{2} & =m_{2}^{2} \sum_{i \geq 1}\left(I_{+\ldots+}^{\bar{\beta}} \zeta_{i}(x)\right)^{2} 2^{-p} \varepsilon(i, 1)^{-p} \\
& =m_{2}^{2} \sum_{i \geq 1, h\left(i_{1}, \ldots, i_{d}\right)=i}\left(I_{+}^{\beta_{1}} \xi_{i_{1}}\left(x_{1}\right) \cdots I_{+}^{\beta_{d}} \xi_{i_{d}}\left(x_{d}\right)\right)^{2} 2^{-p} \kappa(i, 1)^{-p} \\
& =m_{2}^{2} \sum_{i \geq 1, h\left(i_{1}, \ldots, i_{d}\right)=i} \prod_{k=1}^{d}\left(\frac{1}{\Gamma\left(\beta_{k}\right)} \int_{\mathbb{R}}\left(x_{k}-u\right)_{+}^{\beta_{k}-1} \xi_{i_{k}}(u) d u\right)^{2} 2^{-p}\left(1+\frac{i(i-1)}{2}\right)^{-p} \\
& \leq C \sum_{i \geq 1, h\left(i_{1}, \ldots, i_{d}\right)=i} 2^{-p} i^{-p} \prod_{k=1}^{d} i_{k}^{-\beta_{k}+\frac{4}{3}} \leq C \sum_{i_{1}, \ldots, i_{d}} 2^{-p} \prod_{k=1}^{d} i_{k}^{-p-\beta_{k}+\frac{4}{3}} \\
& \leq C \prod_{l=1}^{d} \sum_{i_{k}} 2^{-p} i_{k}^{-p+\frac{4}{3}-\beta_{k}} \\
& <+\infty \text { for } p>2 .
\end{aligned}
$$


Here we use the assumption on $h$ that if $i=h\left(i_{1}, \ldots, i_{d}\right)$, then $i_{k} \leq i$ for $k=1, \ldots, d$. Thus, $\dot{X}^{\bar{\beta}}(x) \in \mathcal{S}^{-p}$ for all $p>2$.

Now, we define the Skorokhod integral for $(\mathcal{S})^{-1}$-valued random fields with respect to $X^{\bar{\beta}}$.

Definition 3.3 Suppose that $F: \mathbb{R}^{d} \longrightarrow(\mathcal{S})^{-1}$ is such that $F(x) \diamond \dot{X}^{\bar{\beta}}(x)$ is $d x$-integrable in $(\mathcal{S})^{-1}$. Then we define the Skorokhod integral of $F$ with respect to $X^{\bar{\beta}}$ by

$$
\delta^{\bar{\beta}}(F):=\int_{\mathbb{R}^{d}} F(x) \delta X^{\bar{\beta}}(x):=\int_{\mathbb{R}^{d}} F(x) \diamond \dot{X}^{\bar{\beta}}(x) d x .
$$

In particular, for Borel sets $A \subset \mathbb{R}^{d}$, we have

$$
\int_{A} F(x) \delta X^{\bar{\beta}}(x):=\int_{\mathbb{R}^{d}} 1_{A}(x) F(x) \diamond \dot{X}^{\bar{\beta}}(x) d x .
$$

Proposition 3.4 If $F(x)=\sum_{\alpha \in \mathcal{J}} c_{\alpha}(x) K_{\alpha}$ for all $x \in \mathbb{R}^{d}$ with

$$
L:=\sup _{\alpha \in \mathcal{J}}\left\{\left\|c_{\alpha}\right\|_{L^{1}\left(\mathbb{R}^{d}\right)}^{2}(2 \mathbb{N})^{-q \alpha}\right\}<+\infty
$$

for some $q \in \mathbb{N}$, then $F$ is integrable with respect to $X^{\bar{\beta}}$, and

$$
\int_{\mathbb{R}^{d}} F(x) \delta X^{\bar{\beta}}(x)=m_{2} \sum_{\alpha \in \mathcal{J}, i \geq 1} \int_{\mathbb{R}^{d}} c_{\alpha}(x)\left(I_{+}^{\bar{\beta}} \zeta_{i}\right)(x) d x K_{\alpha+\varepsilon(i, 1)} .
$$

Proof Since

$$
F(x) \diamond \dot{X}^{\beta}(x)=m_{2} \sum_{\alpha \in \mathcal{J}, i \geq 1} c_{\alpha}(x)\left(I_{+}^{\beta} \zeta_{i}\right)(x) K_{\alpha+\varepsilon(i, 1)}=m_{2} \sum_{\gamma}\left[\sum_{\alpha+\varepsilon(i, 1)=\gamma} c_{\alpha}(x)\left(I_{+}^{\beta} \zeta_{i}\right)(x)\right] K_{\gamma}
$$

by Lemma 2.8 , we wish to show that

$$
M(p)=\sum_{\gamma}\left\|\sum_{\alpha+\varepsilon(i, 1)=\gamma} c_{\alpha}(\cdot)\left(I_{+}^{\beta} \zeta_{i}\right)(\cdot)\right\|_{L^{1}\left(\mathbb{R}^{d}\right)}^{2}(2 \mathbb{N})^{-p \gamma}<+\infty
$$

for some $p>0$. By (3.9) we have

$$
\int_{\mathbb{R}^{d}}\left|c_{\alpha}(x)\left\|\left(I_{+}^{\beta} \zeta_{i}\right)(x) \mid d x \leq C \prod_{k=1}^{d} i_{k}^{\frac{2}{3}-\frac{\beta}{2}}\right\| c_{\alpha} \|_{L^{1}\left(\mathbb{R}^{d}\right)}, \quad \text { where } h\left(i_{1}, \ldots, i_{d}\right)=i\right.
$$

Note that, for all $\gamma, \alpha$, there is at most one $i$ satisfying $\alpha+\varepsilon(i, 1)=\gamma$, and such $i$ is no more than index $(\gamma)$; therefore

$$
\begin{aligned}
& \left\|\sum_{\alpha+\varepsilon(i, 1)=\gamma} c_{\alpha}(\cdot)\left(I_{+}^{\bar{\beta}} \zeta_{i}\right)(\cdot)\right\|_{L^{1}\left(\mathbb{R}^{d}\right)}^{2} \\
& \leq\left(\sum_{\alpha+\varepsilon(i, 1)=\gamma}\left\|c_{\alpha}(\cdot) \prod_{k=1}^{d}\left(I_{+}^{\beta_{k}} \xi_{i_{k}}\right)(\cdot)\right\|_{L^{1}\left(\mathbb{R}^{d}\right)}\right)^{2}
\end{aligned}
$$




$$
\begin{aligned}
& \leq C\left(\sum_{\alpha+\varepsilon(i, 1)=\gamma} \prod_{k=1}^{d} i_{k}^{\frac{2}{3}-\frac{\beta}{2}}\left\|c_{\alpha}\right\|_{L^{1}\left(\mathbb{R}^{d}\right)}\right)^{2} \\
& \leq C\left(\sum_{\alpha+\varepsilon(i, 1)=\gamma}(\operatorname{index}(\gamma))^{\left(\frac{2}{3}-\frac{\beta}{2}\right) d}\left\|c_{\alpha}\right\|_{L^{1}\left(\mathbb{R}^{d}\right)}\right)^{2} \\
& \leq C(\operatorname{index}(\gamma))^{2 d+2} \sum_{\alpha: \exists i \alpha+\varepsilon(i, 1)=\gamma}\left\|c_{\alpha}\right\|_{L^{1}\left(\mathbb{R}^{d}\right)}^{2},
\end{aligned}
$$

where $C$ is a positive constant. Note that index $(\gamma) \leq(2 \mathbb{N})^{\gamma}$, then

$$
\begin{aligned}
M(p) & \leq C \sum_{\alpha, i}\left(\operatorname{index}(\alpha+\varepsilon(i, 1))^{2 d+2}\left\|c_{\alpha}\right\|_{L^{1}(\mathbb{R})}^{2}(2 \mathbb{N})^{-p(\alpha+\varepsilon(i, 1))}\right. \\
& \leq C \sum_{\alpha, i}(2 \mathbb{N})^{(2 d+2)(\alpha+\varepsilon(i, 1))}(2 \mathbb{N})^{-p \varepsilon(i, 1)}(2 \mathbb{N})^{-(p-q) \alpha}\left\|c_{\alpha}\right\|_{L^{1}(\mathbb{R})}^{2}(2 \mathbb{N})^{-q \alpha} \\
& \leq C L \sum_{\alpha}(2 \mathbb{N})^{-(p-q-2 d-2) \alpha} \sum_{i}\left[2\left(1+\frac{i(i-1)}{2}\right)\right]^{-(p-2 d-2)} \\
& <+\infty
\end{aligned}
$$

for $p>q+2 d+2$. Thus,

$$
\begin{aligned}
\int_{\mathbb{R}^{d}} F(x) \delta X^{\beta}(t) & =\int_{\mathbb{R}^{d}} \sum_{\alpha \in \mathcal{J}} c_{\alpha}(x) K_{\alpha} \diamond \sum_{i \geq 1} m_{2}\left(I_{+}^{\bar{\beta}} \zeta_{i}\right)(x) K_{\varepsilon(i, 1)} d x \\
& =m_{2} \sum_{\alpha \in \mathcal{J}, i \geq 1} \int_{\mathbb{R}^{d}} c_{\alpha}(x)\left(I_{+}^{\bar{\beta}} \zeta_{i}\right)(x) d x K_{\alpha+\varepsilon(i, 1)} .
\end{aligned}
$$

Proposition 3.5 Let $F: \mathbb{R}^{d} \longrightarrow(\mathcal{S})^{-1}$ be Skorokhod integrable with respect to $X^{\beta}, Y \in$ $(\mathcal{S})^{-1}$. Then

$$
Y \diamond \delta^{\bar{\beta}}(F)=\delta^{\bar{\beta}}(Y \diamond F)
$$

provided that one side exists.

\section{The stochastic Poisson equation driven by the $d$-parameter fractional Lévy noise}

In this section, we investigate the stochastic Poisson equation driven by the $d$-parameter fractional Lévy noise:

$$
\left\{\begin{array}{l}
\triangle U(x)=-\dot{X}^{\bar{\beta}}(x), \quad x \in D, \\
U(x)=0, \quad x \in \partial D
\end{array}\right.
$$

where $\triangle=\sum_{k=1}^{d} \frac{\partial^{2}}{\partial x_{k}^{2}}$ is the Laplace operator in $\mathbb{R}^{d}, D \subset \mathbb{R}^{d}$ is a given domain with regular boundary, and $\dot{X}^{\bar{\beta}}(x)$ is the $d$-parameter fractional Lévy noise. For example, this equation models the temperature $U(x)$ in $D$ when the boundary temperature is kept equal to 0 and there is a fractional noise heat source in $D$. 
Lemma 4.1 ([10]) Suppose $U$ and $F$ are functions from $D$ in $(4.1)$ to $(\mathcal{S})^{-1}$ such that

$$
\triangle \mathcal{H} U(x, \tau)=\mathcal{H} F(x, \tau), \quad x \in D
$$

for all $(x, \tau) \in D \times K_{q}(R)$ for some $q<+\infty$ and $R>0$.

Furthermore, assume that, for all $j, \frac{\partial^{2}}{\partial x_{j}} \mathcal{H} U(x, \tau)$ is bounded on $(x, \tau) \in D \times K_{q}(R)$, continuous with respect to $x \in D$ for each $\tau \in K_{q}(R)$, and analytic with respect to $\tau \in K_{q}(R)$ for all $x \in D$. Then

$$
\triangle U(x)=F(x), \quad x \in D .
$$

Theorem 4.2 The stochastic Poisson equation (4.1) has a unique continuous solution $U$ : $\bar{D} \longrightarrow(\mathcal{S})^{-1}$. The solution is twice continuously differentiable in $(\mathcal{S})^{-1}$ and takes the form

$$
U(x)=\int_{D} G(x, y) \dot{X}^{\bar{\beta}}(y) d y=m_{2} \sum_{i \geq 1} \int_{D} G(x, y) I_{+\ldots+}^{\bar{\beta}} \zeta_{i}(y) d y K_{\varepsilon(i, 1)}
$$

where $G$ is the classical Green function of $D$ with $G=0$ outside $D$.

Proof Taking the Lévy-Hermite transform of (4.1), we get

$$
\left\{\begin{array}{l}
\Delta u(x, \tau)=-\mathcal{H} \dot{X}^{\bar{\beta}}(x)(\tau), \quad x \in D \\
u(x, \tau)=0, \quad x \in \partial D
\end{array}\right.
$$

where $u(x, \tau)=\mathcal{H} U(x)(\tau)$ and $\mathcal{H} \dot{X}^{\bar{\beta}}(x)(\tau)=m_{2} \sum_{i \geq 1} I_{+\ldots+}^{\bar{\beta}} \zeta_{i}(x) \tau_{\varepsilon(i, 1)}$.

Based on the corresponding solution in the deterministic case, the solution of (4.2) is

$$
u(x, \tau)=\int_{D} G(x, y) \mathcal{H} \dot{X}^{\bar{\beta}}(y)(\tau) d y .
$$

Since $G(x, \cdot) \in L^{1}\left(\mathbb{R}^{d}\right)$, the right-hand side of (4.3) exists for all $\tau \in\left(\mathbb{C}^{\mathbb{N}}\right)_{C}$ and $x \in D$. Hence, $u(x, \tau)$ is defined for such $\tau, x$. Further, by (3.9) we see that

$$
\begin{aligned}
|u(x, \tau)| & \leq m_{2} \sum_{i \geq 1}\left|\tau_{\varepsilon(i, 1)}\right| \int_{D}|G(x, y)|\left|I_{+\ldots+}^{\bar{\beta}} \zeta_{i}(y)\right| d y \\
& \leq C \sum_{i \geq 1}\left|\tau_{\varepsilon(i, 1)}\right| \prod_{k=1, h\left(i_{1}, \ldots, i_{d}\right)=i}^{d} \frac{1}{\Gamma\left(\beta_{k}\right)} i_{k}^{-\frac{\beta_{k}}{2}+\frac{2}{3}} \int_{D}|G(x, y)| d y \\
& \leq C \sum_{i \geq 1}\left|\tau_{\varepsilon(i, 1)}\right| i^{\frac{2}{3} d} \\
& \leq C\left(\sum_{i \geq 1}\left|\tau_{\varepsilon(i, 1)}\right|^{2}(2 \mathbb{N})^{q \varepsilon(i, 1)}\right)^{\frac{1}{2}}\left(\sum_{i \geq 1} i^{\frac{4}{3} d}(2 \mathbb{N})^{-q \varepsilon(i, 1)}\right)^{\frac{1}{2}} \\
& \leq C R\left(\sum_{i \geq 1} i^{\frac{4}{3} d-q}\right)^{\frac{1}{2}}<+\infty
\end{aligned}
$$


for all $\tau \in K_{q}(R)$, where $q>\frac{4}{3} d+1$. Relations (4.3)-(4.4) show that $u(x, \tau)$ is analytical in $\tau$. Thus, we conclude by the characterization theorem (Theorem 2.6) that there exists a function $U: \bar{D} \longrightarrow(\mathcal{S})^{-1}$ such that $u(x, \tau)=\mathcal{H} U(x)(\tau)$. Next, we want to verify the assumptions of Lemma 4.1. It is known from the deterministic PDEs that, for all open and relatively compact $V$ in $D$,

$$
\|u(\cdot, \tau)\|_{C^{2+\alpha}(V)} \leq C\left(\|\Delta u(\cdot, \tau)\|_{C^{\alpha}(V)}+\|u(\cdot, \tau)\|_{C(V)}\right)
$$

for all $\tau \in\left(\mathbb{C}^{\mathbb{N}}\right)_{C}$ (see [2]). Since $\Delta u(x, \tau)=-\mathcal{H} \dot{X}_{x}^{\bar{\beta}}(\tau)$ and $u$ are bounded on $D \times K_{q}(R)$, it follows that $\frac{\partial^{2}}{\partial x_{j}} u(x, \tau)$ is bounded for such $x, \tau$. Thus, by Lemma 4.1, $U$ is a solution of (4.1).

Further, by the proof of Theorem 3.2 and the fact that $G(x, \cdot) \in L^{1}\left(\mathbb{R}^{d}\right)$, for $p>1$, we have

$$
\|U(x)\|_{-p} \leq \int_{D}\left\|\dot{X}_{y}^{\bar{\beta}}\right\|_{-p}|G(x, y)| d y<+\infty
$$

that is, the Bochner integral $\int_{D} G(x, y) \dot{X}_{y}^{\bar{\beta}} d y$ exists in $(\mathcal{S})^{-1}$, and

$$
\int_{D} G(x, y) \dot{X}_{y}^{\bar{\beta}} d y=m_{2} \sum_{i \geq 1} \int_{D} G(x, y) I_{+\ldots+}^{\bar{\beta}} \zeta_{i}(x) K_{\varepsilon(i, 1)} .
$$

Then the right-hand side of (4.3) is the Lévy-Hermite transform of (4.6). Thus, we finish the proof of the theorem.

Remark The solution $U(x)$ is a strong solution in $(\mathcal{S})^{-1}$, that is, for each $x$,

$$
\langle\langle U(x), f\rangle\rangle=\int_{D} G(x, y)\left\langle\left\langle\dot{X}_{y}^{\bar{\beta}}, f\right\rangle\right\rangle d y, \quad \forall f \in(\mathcal{S}) .
$$

\section{The stochastic Schrödinger equation driven by the $d$-parameter fractional Lévy noise}

In this section, we investigate the stochastic Schrödinger equation driven by the $d$ parameter fractional Lévy noise:

$$
\left\{\begin{array}{l}
\frac{1}{2} \triangle U(x)+V(x) \diamond U(x)=-f(x), \quad x \in D, \\
U(x)=0, \quad x \in \partial D
\end{array}\right.
$$

where $\Delta=\sum_{k=1}^{d} \frac{\partial^{2}}{\partial x_{k}^{2}}$ is the Laplace operator in $\mathbb{R}^{d}, D$ is a given bounded domain in $\mathbb{R}^{d}$, and $V(x)$ and $f(x)$ are given stochastic distribution-valued processes. We replace the potential $V(x)$ proportional to the $d$-parameter fractional Lévy noise $\dot{X}^{\bar{\beta}}(x)$, that is,

$$
\left\{\begin{array}{l}
\frac{1}{2} \Delta U(x)+\rho \dot{X}^{\bar{\beta}}(x) \diamond U(x)=-f(x), \quad x \in D \\
U(x)=0, \quad x \in \partial D
\end{array}\right.
$$

where $\rho \in \mathbb{R}$ is a constant. If $\rho>0$, then this case is called the attractive case. 
Let $\lambda_{0}$ be the smallest eigenvalue for the operator $-\frac{1}{2} \Delta$ in $\mathbb{R}^{d}$, that is, $\lambda_{0}$ is the smallest $\lambda$ such that the boundary value problem

$$
\left\{\begin{array}{l}
\frac{1}{2} \triangle u(x)=\lambda u(x), \quad x \in D \\
u(x)=0, \quad x \in \partial_{R} D
\end{array}\right.
$$

where $\partial_{R} D$ is the regular boundary of $D$, has a solution $u \in C^{2}(D)$.

Let $\left\{b_{t}, t \geq 0\right\}$ denote an auxiliary Brownian motion independent of $X$, and let $\widehat{E}^{x}$ denote the expectation with respect to the law $\widehat{P}^{x}$ of $\left\{b_{t}, t \geq 0\right\}$ starting at $x$. Define the first exit time $\tau_{D}$ for $b_{t}$ from $D$ by

$$
\tau_{D}=\inf \left\{t>0: b_{t} \bar{\in} \overline{\}}\right.
$$

In fact,

$$
\lambda_{0}=\sup \left\{\rho \in \mathbb{R}: \widehat{E}^{x}\left[\exp \left[\rho \tau_{D}\right]\right]<+\infty\right\}, \quad \forall x \in D .
$$

(See, e.g., Durrett[3].)

Theorem 5.1 Suppose that $f(x)$ is a stochastic distribution process such that $\widetilde{f}(x, \tau)=$ $\mathcal{H} f(x)(\tau)$ is bounded for $(x, z) \in D \times K_{q}(R)$ with some $q$, R. Let $D$ be a bounded domain in $\mathbb{R}^{d}$ with all its points regular for the classical Dirichlet problem in $D$. Let $\rho<\lambda_{0}$ be a constant. Then, there exist a unique $(\mathcal{S})^{-1}$ solution $U(x)$ of $(5.2)$ expressed as

$$
U(x)=\widehat{E}^{x}\left[\int_{0}^{\tau_{D}} \exp ^{\diamond}\left[\rho \int_{0}^{t} \exp ^{\diamond}\left(\dot{X}^{\bar{\beta}}\left(b_{s}\right)\right) d s\right] \diamond f\left(b_{t}\right) d t\right] .
$$

Proof Choose $\tau \in\left(\mathbb{C}^{\mathbb{N}}\right)_{C}$. By taking the Lévy-Hermite transform of (5.2) we get

$$
\left\{\begin{array}{l}
\frac{1}{2} \Delta u(x, \tau)+\rho\left(\mathcal{H} \dot{X}^{\bar{\beta}}\right)(x)(\tau) \diamond u(x, \tau)=-\widetilde{f}(x, \tau), \quad x \in D, \\
U(x)=0, \quad x \in \partial D
\end{array}\right.
$$

where $u(x, \tau)=\mathcal{H} U(x)(\tau),\left(\mathcal{H} \dot{X}^{\bar{\beta}}(x)\right)(\tau)=m_{2} \sum_{i \geq 1} I_{+\cdots+} \bar{\beta} \zeta_{i}(x) \tau_{\varepsilon(i, 1)}$. Then, by a complex version of the Feynman-Kac formula we get the unique solution of (5.5)

$$
u(x, \tau)=\widehat{E}^{x}\left[\int_{0}^{\tau_{D}} \exp \left[\rho \int_{0}^{t} \exp \left(\mathcal{H} \dot{X}^{\bar{\beta}}\left(b_{s}\right)(\tau)\right) d s\right] \tilde{f}\left(b_{t}, \tau\right) d t\right],
$$

provided that the expression converges. Note that

$$
\begin{aligned}
\left|\left(\mathcal{H} \dot{X}^{\bar{\beta}}\left(b_{s}\right)\right)(\tau)\right|^{2} & =m_{2}^{2}\left|\sum_{i \geq 1} I_{+\ldots+}^{\bar{\beta}} \zeta_{i}\left(b_{s}\right) \tau_{\varepsilon(i, 1)}\right|^{2} \\
& \leq m_{2}^{2}\left(\sum_{i \geq 1} \sup _{x}\left|I_{+\ldots+}^{\bar{\beta}} \zeta_{i}(x)\right|\left|\tau_{\varepsilon(i, 1)}\right|\right)^{2} \\
& \leq C m_{2}^{2}\left(\sum_{i \geq 1} i^{\frac{2}{3} d-\sum_{i=1}^{d} \frac{\beta_{i}}{2}}\left|\tau_{\varepsilon(i, 1)}\right|\right)^{2}
\end{aligned}
$$




$$
\begin{aligned}
& \leq \mathrm{Cm}_{2}^{2} \sum_{i \geq 1} i^{\frac{4}{3} d-\sum_{i=1}^{d} \beta_{i}}(2 \mathbb{N})^{-q \varepsilon(i, 1)} \sum_{i \geq 1}\left|\tau_{\varepsilon(i, 1)}\right|(2 \mathbb{N})^{q \varepsilon(i, 1)} \\
& \leq C m_{2}^{2} \sum_{i \geq 1} i^{\frac{4}{3} d-\sum_{i=1}^{d} \beta_{i}-q} \sum_{\alpha}\left|\tau_{\varepsilon(i, 1)}\right|(2 \mathbb{N})^{q \alpha}:=C(q, R)^{2}<+\infty
\end{aligned}
$$

for all $\tau \in K_{q}(R)$ and $q>\frac{4}{3} d-\sum_{i=1}^{d} \beta_{i}+1$. Therefore, by the assumption on $f$, for $\tau \in K_{q}(R)$, we have

$$
\begin{aligned}
|u(x, \tau)| & \leq M \widehat{E}^{x}\left[\int_{0}^{\tau_{D}} \exp \left[\rho \int_{0}^{t} \exp C(q, R) d s\right] d t\right] \\
& \leq M \widehat{E}^{x}\left[\int_{0}^{\tau_{D}} \exp [\rho t \exp C(q, R)] d t\right] \\
& \leq \frac{M}{\rho \exp C(q, R)} \widehat{E}^{x}\left[\exp \left[\rho \tau_{D} \exp C(q, R)\right]\right],
\end{aligned}
$$

where $M=\sup \left\{\tilde{f}(x, \tau),(x, \tau) \in D \times K_{q_{1}}\left(R_{1}\right)\right\}$.

Now choose $q_{2}, R_{2}$, and $\varepsilon>0$ such that

$$
\rho\left[\exp C\left(q_{2}, R_{2}\right)\right]<(1-\varepsilon) \lambda_{0} .
$$

Then, for $q>\max \left\{q_{1}, q_{2}\right\}$ and $R<\min \left\{R_{1}, R_{2}\right\}$, we have

$$
|u(x, \tau)|<\frac{M}{\rho \exp C(q, R)} \widehat{E}^{x}\left[\exp \left[(1-\varepsilon) \lambda_{0} \tau_{D}\right]\right]<+\infty
$$

for all $\tau \in K_{q}(R)$.

Since $u(x, \tau)$ is analytical in $\tau \in K_{q}(R)$, we conclude by the characterization theorem (Theorem 2.6) that there exists a function $U: \bar{D} \longrightarrow(\mathcal{S})^{-1}$ such that $u(x, \tau)=\mathcal{H} U(x)(\tau)$. Next, we want to verify the assumptions of Theorem 2.9. It is known from the deterministic PDEs that, for all open and relatively compact $V$ in $D$,

$$
\|u(\cdot, \tau)\|_{C^{2+\alpha}(V)} \leq C\left(\|\Delta u(\cdot, z)\|_{C^{\alpha}(V)}+\|u(\cdot, \tau)\|_{C(V)}\right)
$$

for all $\tau \in\left(\mathbb{C}^{\mathbb{N}}\right)_{C}$. It follows that $\frac{\partial^{2}}{\partial x_{j}} u(x, \tau)$ is uniformly bounded for such $(x, \tau) \in V \times K_{q}(R)$ for each open set $V \Subset D$. Thus, by Theorem 2.9, $U$ is a solution of (5.1). Moreover, we can verify directly that the right-hand side of (5.6) is the Lévy-Hermite transform of (5.4). Thus, we finish the proof of the theorem.

Remark The solution $U(x)$ is a strong solution in $(\mathcal{S})^{-1}$, that is, for each $x$,

$$
\left\{\begin{array}{l}
\frac{1}{2} \triangle\langle\langle U(x), f\rangle\rangle+\left\langle\left\langle\rho \dot{X}^{\bar{\beta}}(x) \diamond U(x), f\right\rangle\right\rangle=-\langle\langle f(x), f\rangle\rangle, \quad x \in D \\
\langle\langle U(x), f\rangle\rangle=0, \quad x \in \partial D,
\end{array}\right.
$$


Competing interests

The authors declare that they have no competing interests.

\section{Authors' contributions}

The authors have made the same contribution. Both authors read and approved the final manuscript.

\section{Author details}

'Department of Applied Mathematics, School of Physical and Mathematical Sciences, Nanjing Tech University, Nanjing,

P.R. China. ${ }^{2}$ Department of Mathematics, Nanjing University, Nanjing, P.R. China.

\section{Publisher's Note}

Springer Nature remains neutral with regard to jurisdictional claims in published maps and institutional affiliations.

Received: 23 May 2018 Accepted: 23 October 2018 Published online: 16 November 2018

\section{References}

1. Bender, C., Marquardt, T.: Stochastic calculus for convoluted Lévy processes. Bernoulli 14(2), 499-518 (2008)

2. Bers, L., John, F., Schechter, M.: Partial Differential Equations, Interscience (1964)

3. Durrett, R.: Brownian Motion and Martingales in Analysis. Wadsworth, Belmont (1984)

4. Elliott, R.C., Van der Hoek, J.: A general fractional white noise theory and applications to finance. Math. Finance 13 301-330 (2003)

5. Holden, H., Oksendal, B., Uboe, J., Zhang, T.: Stochastic Partial Differential Equations: a modeling, white noise functional approach, 2nd edn. Springer, (2010)

6. Huang, Z., Li, C.: On fractional stable processes and sheets: white noise approach. J. Math. Anal. Appl. 325, 624-635 (2007)

7. Huang, Z., Li, P.: Generalized fractional Lévy processes: a white noise approach. Stoch. Dyn. 6, 473-485 (2006)

8. Huang, Z., Li, P.: Fractional generalized Lévy random fields as white noise functionals. Front. Math. China 2, 211-226 (2007)

9. Huang, Z., Lü, X., Wan, J.: Fractional Lévy processes and noises on Gel'fand triple. Stoch. Dyn. 10, 37-51 (2010)

10. Lokka, A., Oksendal, B., Proske, F.: Stochastic partial differential equations driven by Lévy space-time white noise. Ann. Appl. Probab. 14(3), 1506-1528 (2004)

11. Lü, X., Dai, W.: White noise analysis for fractional Lévy processes and its applications. (to appear)

12. Lü, X., Huang, Z., Dai, W.: Generalized fractional Lévy random fields on Gel'fand triple: a white noise approach. Front. Math. China 6, 493-506 (2011)

13. Lü, X., Huang, Z., Wan, J.: Fractional Lévy processes on Gel'fand triple and stochastic integration. Front. Math. China 3, 287-303 (2008)

14. Marquardt, T.: Fractional Lévy processes with an application to long memory moving average processes. Bernoulli 12 , 1099-1126 (2006)

15. Nualart, D., Schoutens, W.: Chaotic and predictable representations for Lévy processes. Stoch. Process. Appl. 90(1), 109-122 (2000)

16. Samko, S.G., Kilbas, A.A., Marichev, O.I.: Fractional Integrals and Derivatives: Theory and Applications. Gordon \& Breach, New York (1987)

\section{Submit your manuscript to a SpringerOpen ${ }^{\circ}$ journal and benefit from:}

- Convenient online submission

- Rigorous peer review

- Open access: articles freely available online

- High visibility within the field

- Retaining the copyright to your article

Submit your next manuscript at $\gg$ springeropen.com 